\title{
Stability and Change in the Educational Gradient of Divorce. A Comparison of Seventeen Countries
}

\author{
Juho Härkönen and Jaap Dronkers
}

In a series of papers, William J. Goode argued that the relationship between modernization and the class composition of divorce is inverse. Starting from his hypothesis, we examine the relationship between female education and the risk of divorce over time in 17 countries. We expect that the relationship differs across countries and across time, so that women with higher education have a higher risk of divorce in countries and at times when the social and economic costs of divorce are high, and that there is no relationship or a negative relationship where these costs are lower. Using discrete-time event-history techniques on data on first marriages from the Fertility and Family Surveys (FFS), we find that women with higher education had a higher risk of divorce in France, Greece, Italy, Poland, and Spain. We do not find a relationship between education and divorce in Estonia, Finland, West-Germany, Hungary, Latvia, Sweden, and Switzerland, nor, depending on the model specification, in Flanders and Norway. In Austria, Lithuania, and the United States, the educational gradient of divorce is negative. Furthermore, as predicted by our hypotheses, the educational gradient becomes increasingly negative in Flanders, Finland, France, Hungary, Italy, Lithuania, Poland, Sweden, and the United States. We explore this variation across time and countries in more detail with multilevel models and direct measures on the legal, social, and economic environment of the countries. We find that the de-institutionalization of marriage and unconventional family practices are associated with a negative educational gradient of divorce, while welfare state expenditure is associated with a more positive gradient.

\section{Introduction}

The increase in divorce is one of the most visible changes in family life in Western countries. Despite this general trend, divorce is more common not only in some countries but also in some social groups. These social differences reflect social inequalities in marital life and in the (mainly negative) consequences of divorce. However, these differences are not necessarily stable across time and space. For example, the current American experience of the lower class character of single parenthood does not characterize all earlier periods or all countries (McLanahan, 2004). Despite these findings, studies on divorce risk factors usually start from the assumption 
that the effects of these are constant across time and across societies, even though there are good theoretical reasons to expect otherwise (Teachman, 2002: 332).

William J. Goode was probably the first to argue a link between societal factors and the social composition of divorce. In a series of papers (Goode, 1962, 1970, 1993), he suggested that the relationship between the social composition of divorce and the level of modernization was inverse. He expected that the once positive relationship between social status and divorce-characteristic of the early stages of modernization with high legal, social, and economic barriers to divorce-would gradually fade away as these barriers did. In the end, divorce would be more common in the lower classes, generally accompanied by more marital strain. In the same way, one can also expect cross-national differences in the social structure of divorce, depending on the social, legal, and economic environment of family life.

In this article, we examine the effects of female education on divorce over time in 17 countries [Austria, Estonia, Finland, Flanders (Belgium), France, West Germany, Greece, Hungary, Italy, Latvia, Lithuania, Norway, Poland, Spain, Sweden, Switzerland, and United States]. Starting from Goode's hypothesis, we ask (i) whether there are cross-country differences in the educational gradient of divorce, (ii) whether the effect of education on divorce has become more negative, and (iii) whether the differences between countries and across time can be linked to macro-level variables reflecting the legal and socio-economic environment of family life. With data from the Fertility and Family Surveys (FFS), we first establish the stabilities and changes by performing discrete-time event-history analyses in each country and then continue with multilevel discrete-time event-history analyses to look for explanations for the patterns found.

\section{Female Education and Divorce: Theoretical Perspectives}

The best-known hypothesis concerning the effects of female education on divorce is found in Gary Becker's (1981) economic theory of the family. Becker argued that because education improves women's chances in the labour market and thus their economic independence, but decreases the economic gains of marriage, women with more education will divorce more easily. Women with higher education might also hold more liberal values (Levinger, 1976), or have better resources for handling the divorce process (Blossfeld et al., 1995), thus being more prone to end an unsatisfactory relationship.
Other accounts, however, lead to opposite predictions. It has been argued that education improves resources-such as social, cultural, economic, and cognitive skills- that increase the stability of relationships, either by successful partner matching or by enhancing communication skills and other factors that make a relationship work (Amato, 1996; Hoem, 1997; Ono, 1998; Dronkers, 2002). In line with the original Goode hypothesis, we can also assume that those in the lower social strata have more marital strain because of greater socio-economic hardship (also Hoem, 1997; Oppenheimer, 1997; Jalovaara, 2003). In addition, as women's economic resources have become increasingly important for the status of the whole family (Oppenheimer, 1997), men have increasingly more to loose from divorcing highly educated women.

Despite the opposing predictions made by these hypotheses, all these theories share an assumption that marriages are maintained as long as the well-being of the partner(s) exceeds that of divorce (cf. Teachman, 2002: 331-332). The main difference between the theories is in the mechanisms (economic, social, cultural, and cognitive) emphasized. There seems to be no a priori reason to rule out the possibility that these mechanisms operate simultaneously, even if in opposing directions. The resulting effect of female education on divorce thus depends on the balance of these different mechanisms.

\section{Cross-Country and Period Differences in the Effects of Female Education?}

Which socio-economic variables affect the balance between the mechanisms discussed above and thus influence the educational gradient of divorce? Following the general costs and benefits approach outlined above, one can point to various legal, social, and economic factors, which affect both the costs of divorce and the benefits of marriage.

An obvious factor is divorce legislation. The effect of the liberalization in divorce laws on divorce rates has been a topic of great interest, and results suggest that the liberalization of legislation increases divorce rates, at least in the short run (Friedberg, 1998; Wolfers, 2003). The strictness of divorce laws may not, however, have equal effects on all marriages. Goode (1970: 85-86) hypothesized that strict divorce laws would mainly suppress the chances of divorce among the lower classes, while the upper classes would be more resourceful in finding ways to get around strict rules. Goode went on 
to argue that with more liberal laws, 'the normal difficulties of lower-class family life were permitted an expression in divorce' (Goode, 1970: 85-86).

Social norms and conventions surrounding family life can produce similar differences. First, strict social norms against the dissolution of a union may require extra resources (such as high levels of education) to overcome them, whereas loosened norms of marital life reduce the importance of such resources. Second, if divorce is relatively rare in a society, such behaviour is more innovative, thus, again, requiring more resources. Later, through social learning, such behaviour can spread to the wider population and the lower classes (Chan and Halpin, 2005).

The economic environment, including the welfare state, is apparently important in shaping the relative costs and benefits of divorce. First, good female labour market possibilities decrease women's dependency on their husbands. When female participation in the labour market is acceptable and widespread, even women with lower levels of human capital have better chances for economic independence. The welfare state can have similar effects: with extensive and generous welfare benefits and services, women can support themselves and their children independently, either by working or through benefits (cf. Orloff, 1993). Here again, the effect may be more pronounced among lesseducated women. On the contrary, welfare states may also reduce economic strain (cf. Hoem, 1997; Oppenheimer, 1997; Jalovaara, 2003), thus stabilizing marriages among the lower classes.

\section{Previous Results and Hypotheses}

What is the empirical evidence regarding the effects of female education on divorce? In general, the results are mixed. American, Nordic, and British studies generally find a negative effect (e.g. Bumpass and Sweet, 1972; Hoem, 1997; Berrington and Diamond, 1999; Teachman, 2002; Jalovaara, 2003; Lyngstad, 2004). Other studies, however, claim different results. German and Dutch research has generally reported a positive effect (e.g. Diekmann and Klein, 1991; Kalmijn et al., 2004). Support for cross-national differences is also provided by Blossfeld and colleagues (1995), who found the strongest positive effects in Italy, followed by Germany, and then Sweden. Comparative differences were also found in the earlier cross-national case studies-Chester's Divorce in Europe (1977).
Few studies have explicitly tested for change in the effect of female education on union disruption. Again, the results from these few studies have given conflicting results. Martin and Bumpass (1989) found that the effect had generally become more negative in the younger American marriage cohorts, whereas Teachman's (2002) results pointed to stability in the educational gradient of divorce. Härkönen (2005) reported a similar result for Finland. However, the findings of Raley and Bumpass (2003) do seem to suggest that union disruption has increased among the lower groups in the United States but stayed stable in the upper ones. For the United Kingdom, Chan and Halpin (2005) found that the relationship between female education and divorce has changed from a positive to a negative one, and similar changes were also found in Sweden by Hoem (1997).

These results send conflicting messages. On the one hand, the cross-country (and some cross-cohort) differences suggest that societal-level factors do have an effect, as suggested by the theoretical discussion above. On the other, one may expect more consistent trends across countries, following from the profound changes in all post-war Western societies. Different results could be attributable to different model specifications. Therefore, to draw better conclusions, more consistent analyses with comparable data and models are needed.

Based on the theoretical discussion and previous research, we formulate three hypotheses.

- Hypothesis 1: The effect of education on divorce risk varies across countries.

- Hypothesis 2: The effect of education on divorce risk becomes more negative, that is, the divorce risk of less-educated women increases relative to women with higher educational levels.

- Hypothesis 3: Lower legal, social, and economic costs of divorce increase the divorce risk of less-educated women relative to better-educated women, thus making the effect of education on divorce more negative.

\section{Data and Methods}

\section{FFS}

We use data for our 17 countries from the FFS, collected by the Population Activities Unit of the United Nations Economic Commission for Europe (see Andersson and Philipov, 2002). The FFS is a retrospective survey, which includes information on the fertility, family, education, and occupational histories of those interviewed. The data were collected between 1989 and 1999, in different 
years in different countries. We selected the first marriages of women who reported having been married ${ }^{1}$ and who were aged 17 years or more at the time of their first marriage. To be suitable for event-history analysis in discrete time, we re-organized the data into personyear form (Yamaguchi, 1991). After considerable data cleaning, we ended up with a sample of 44,229 women, 511,714 person-years, and 7,978 divorces in 17 countries (see Table 1). ${ }^{2}$ Our dependent variable is divorce, which was coded 1 if the marriage dissolved at a particular year and 0 otherwise.

Our main independent variable is educational attainment, which was coded into three categories, according to the International Standard Classification of Education (ISCED) scheme: low (0-2), middle (3), and high (4-6) (see Table 1). Our education variable measures the educational attainment of the respondent at the time of interview. Although not an optimal measure for our purposes, this was decided on after even more serious problems were discovered as to other possible measures. For example, educational histories were missing for a large number of our countries. The same problems prevented the use of years of education as well.

As controls, we include the linear and squared terms of marital duration, age at marriage, parental divorce, premarital cohabitation and pre-marital children (see Table 1). We lacked information on parental divorce for the Norwegian data, and on cohabitation for the Estonian data. In the first event-history analyses, we use the year of marriage to measure the changing social environment of union disruption. ${ }^{3}$ However, because we include duration (linear and quadratic terms) in the models, and as cohort plus duration equals period, the effects of the year of marriage can be interpreted as either cohort or period effects (see Allison, 1995: 142-143; Teachman, 2002). Following Teachman (2002), we interpret the marriage cohort variables as capturing the period effect of a change in the social context of family life. ${ }^{4,5}$ This interpretation is supported by the large body of literature pointing to the importance of period effects over cohort effects (Thornton and Rodgers, 1987; Lutz et al., 1991).

\section{Macro-Variables}

To analyse the effects of the societal environment in shaping the costs of divorce, we collected data on divorce legislation, social policies, the value environment, family practices, and the 'general level of modernization' (see Table 1; for a more detailed description, see Härkönen and Dronkers, 2006). The time-dependent nature of the variables varied. For some measures (for instance divorce legislation, extra-marital births, female economic activity, and urbanization levels), we were able to find data for relatively long time periods. For others (such as value measures), we had to restrict ourselves to a few time-points, or even to only one. In general, with the exception of divorce legislation, one decade was the basic unit of time used. The averages and standard deviations are given in Table 1, based on person-year categories, both total and per country. If the standard deviation of a macro-variable is 0 , that variable is not time dependent but varies between countries only. A standard deviation of more than 0 means that the variable is time dependent, or that we found data for only one point in time. The case of divorce legislation is the only instance where the lack of variation in time reflects the stability of the divorce law of a country and not a lack of data.

\section{Divorce legislation}

The strictness of divorce legislation is measured with a single time-dependent variable. During the period of study, divorce legislation varied considerably, from prohibition of divorce to unilateral no-fault divorce. We use a three-fold categorization as our divorce law measure (cf. Glendon, 1987; Castles and Flood, 1993):

(i) Divorce not permitted (a) or permitted on the grounds of fault or other major disruption of marital life (b). The institutionalization of marriage remains the leading principle, and the divorce process (if permitted) is difficult and lengthy.

(ii) Divorce permitted, possibly alongside (b), on the mutual consent of the spouses, prolonged separation, other measures of factual breakdown of marriage, or other less-restrictive legislation. Shows more understanding for the will of the spouses.

(iii) No (or very minor) judicial grounds to deny divorce: unilateral non-fault divorce granted on the basis of the will of either spouse with very short waiting or 'reconsideration' times.

We joined (a) and (b) into a common category, because the small number of events in (a) did not permit sustainable analysis. This is also used as the reference category.

Data on divorce laws were collected from Boschan (1972), Chester (1977), Chloros (1978), Lobodzinska (1982), Moskoff (1983), Glendon (1987, 1989), Goode (1993), Nakonezny et al. (1995), Friedberg (1998), and Hamilton and Perry (2002). In some cases, classifying a 


\begin{tabular}{|c|c|c|c|c|c|c|c|c|c|c|c|}
\hline 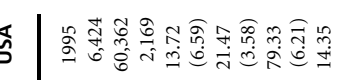 & $\underset{\substack{\infty \\
\infty}}{\infty}$ & $\stackrel{\vec{N}}{\stackrel{4}{*}}$ & 芦 & 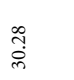 & 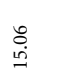 & 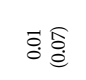 & 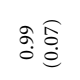 & 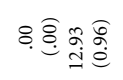 & $\stackrel{\ln }{i} \stackrel{\infty}{=}$ & $\begin{array}{ll}\infty \\
\infty \\
\infty\end{array}$ & $\hat{e}$ \\
\hline 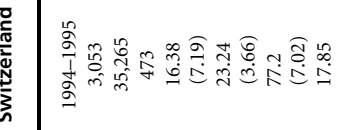 & $\stackrel{\text { in }}{\rightarrow}$ & $\stackrel{n}{\stackrel{0}{\leftrightarrows}}$ & $\stackrel{\infty}{\infty}$ & 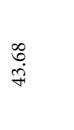 & $\stackrel{\infty}{\stackrel{\circ}{~}}$ & छุ & ฮุ & 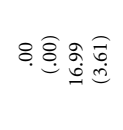 & 욜 & $\stackrel{\substack{\infty \\
\stackrel{\infty}{e}}}{a}$ & 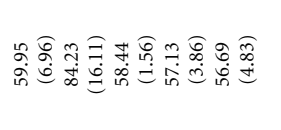 \\
\hline 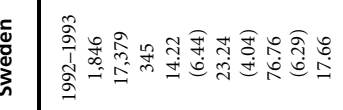 & \begin{tabular}{l}
8 \\
\hdashline \\
\hdashline
\end{tabular} & $\begin{array}{l}\infty \\
\stackrel{\infty}{0} \\
\stackrel{\infty}{\infty}\end{array}$ & 总 & $\underset{\substack{2 \\
\infty}}{\infty}$ & 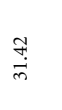 & ৪) & : & 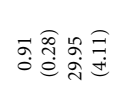 & 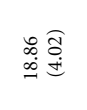 & 虍 & 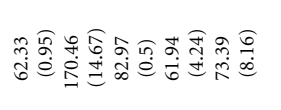 \\
\hline 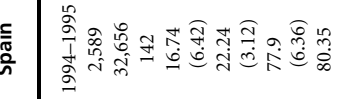 & $\stackrel{\widetilde{2}}{=}$ & $\stackrel{n}{2}$ & \% & in & 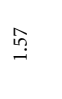 & 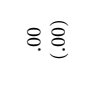 & 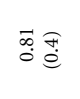 & 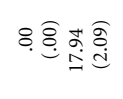 & 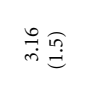 & 露 & 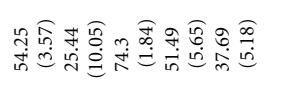 \\
\hline 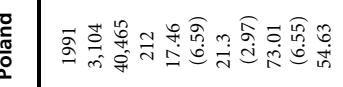 & $\begin{array}{ll}6 \\
6 \\
0\end{array}$ & $\stackrel{\infty}{\stackrel{\infty}{ \pm ்}}$ & 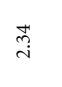 & $\ddot{\dot{m}}$ & $\hat{\mathrm{j}}$ & ๖: & $\underset{\mathrm{g}}{\mathrm{g}}$ & \&. & $\stackrel{\infty}{\widehat{g}}$ & 㕍 & 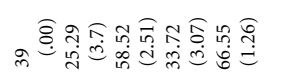 \\
\hline 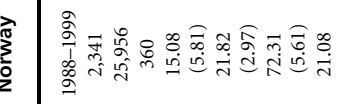 & $\stackrel{\vec{\infty}}{\overrightarrow{+}}$ & $\underset{m}{\vec{i}}$ & 1 & 品 & 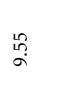 & ঃ. & ọ & 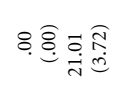 & 虎 & 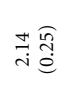 & 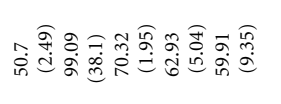 \\
\hline 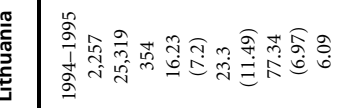 & $\stackrel{\infty}{\infty}$ & 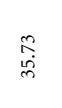 & $\stackrel{\stackrel{\leftrightarrow}{g}}{\stackrel{\sim}{g}}$ & $\stackrel{\substack{h \\
=}}{=}$ & $\stackrel{\infty}{i}$ & ৪. & $\underset{-\sigma}{\sigma}$ & 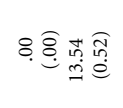 & 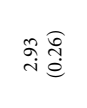 & 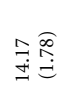 & 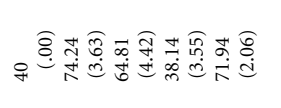 \\
\hline 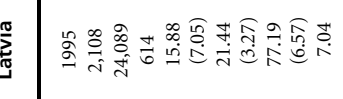 & : & $\stackrel{\text { ì }}{\hat{n}}$ & $\stackrel{2}{\circ}$ & 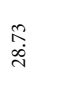 & $\stackrel{े}{\overrightarrow{+}}$ & ৪. & $\stackrel{\mathrm{Q}}{=}$ & 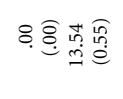 & 구영 & 亦兽 & 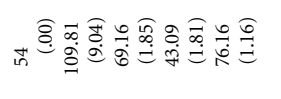 \\
\hline 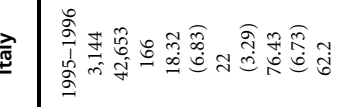 & y. & $\underset{i}{\stackrel{F}{i}}$ & $\stackrel{\text { gे }}{i}$ & $\stackrel{m}{m}$ & $\stackrel{\Re}{i}$ & 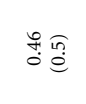 & 종 & ৪) & $+\underset{\infty}{+\frac{\Im}{i}}$ & 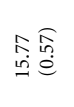 & 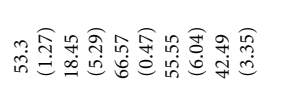 \\
\hline 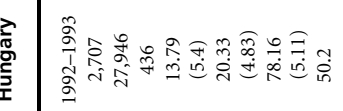 & 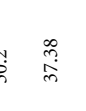 & I & 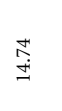 & $\stackrel{\infty}{\stackrel{\infty}{\infty}}$ & $\stackrel{\vec{\sigma}}{\dot{m}}$ & 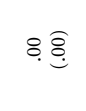 & $\underset{\Xi}{\stackrel{g}{g}}$ & 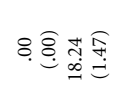 & $\sqrt[4]{-i}$ & 藏 & 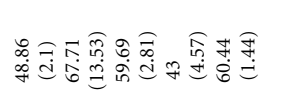 \\
\hline 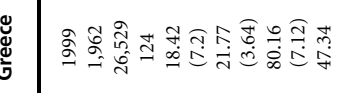 & $\stackrel{m}{?}$ & 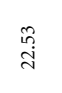 & $\stackrel{\text { oे }}{i}$ & $\underset{\infty}{\infty}$ & $\underset{\text { q }}{\mathrm{q}}$ & $\stackrel{\infty}{0}$ & 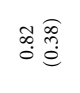 & 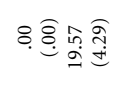 & 离 & 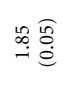 & 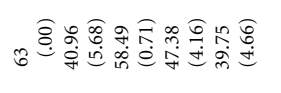 \\
\hline 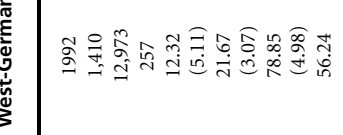 & d & $\stackrel{2}{2}$ & : & $\begin{array}{c}\hat{b} \\
\substack{\infty \\
\infty}\end{array}$ & $\underset{\infty}{\stackrel{9}{\infty}}$ & : & 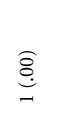 & 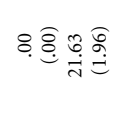 & 空 & 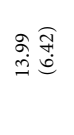 & 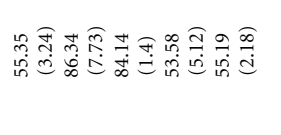 \\
\hline 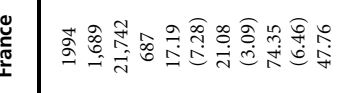 & $\stackrel{1}{\circ}$ & 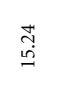 & $\bar{a}$ & बें & 藏 & 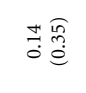 & $\stackrel{\substack{n \\
\hdashline}}{\infty}$ & 圶 & 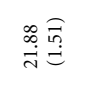 & $\stackrel{\stackrel{\infty}{\infty}}{\stackrel{\leftrightarrow}{\leftrightarrows}}$ & 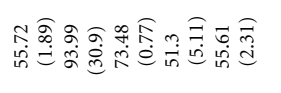 \\
\hline 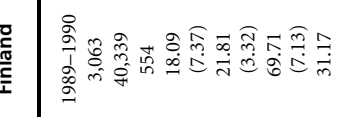 & i̊ & 官 & $\tilde{n}$ & $\begin{array}{l}\overrightarrow{0} \\
\dot{m} \\
\dot{m}\end{array}$ & $\stackrel{\substack{+\dot{f}}}{2}$ & ৪: & 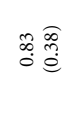 & 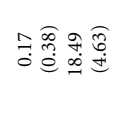 & 拿哭 & 站怘 & 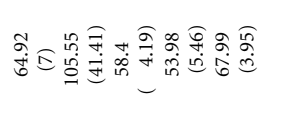 \\
\hline 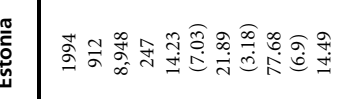 & की & $\overrightarrow{\underline{m}}$ & $\stackrel{m}{\mathrm{i}}$ & 1 & $\underset{\dot{+}}{\stackrel{+}{+}}$ & ৪ลํ. & $\stackrel{\widehat{0}}{\stackrel{0}{0}}$ & ৪) & 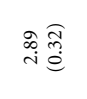 & 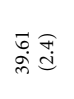 & 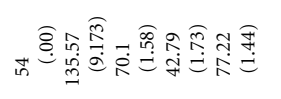 \\
\hline 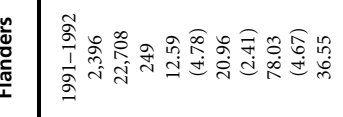 & $\overbrace{\substack{n \\
0}}^{0}$ & త్రై & :̊n & $\underset{7}{\stackrel{7}{n}}$ & $\stackrel{\leftrightarrows}{\leftrightarrows}$ & : & 括 & ৪) & 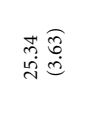 & 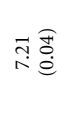 & 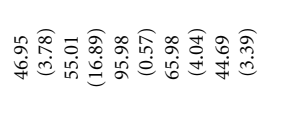 \\
\hline 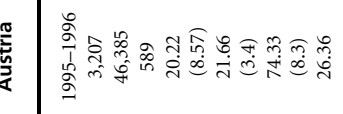 & $\overbrace{\substack{0 \\
i}}^{\circ}$ & $\stackrel{\infty}{\stackrel{\infty}{=}}$ & $\begin{array}{l}\infty \\
\substack{\infty \\
\infty} \\
\infty\end{array}$ & 点 & 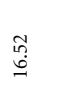 & ৪) & อ & 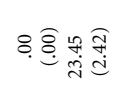 & 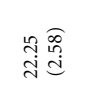 & $\rightarrow \stackrel{\leftrightarrow}{\leftrightarrows}$ & 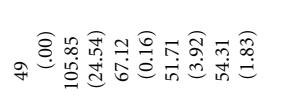 \\
\hline 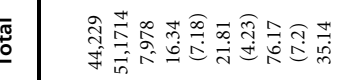 & 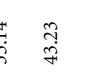 & 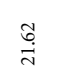 & ڤn & 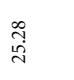 & $\therefore$ & o̊ & 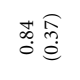 & 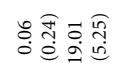 & 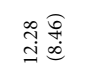 & 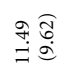 & 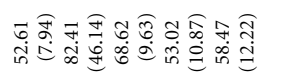 \\
\hline 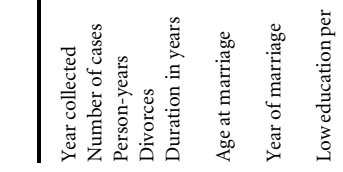 & 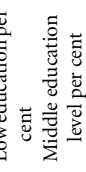 & & & 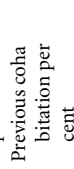 & 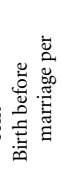 & $\frac{\pi}{9}$ & & 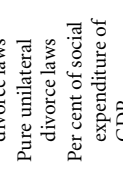 & 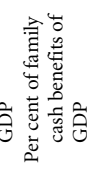 & $\vec{\sigma}$ & 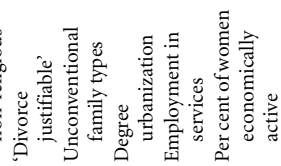 \\
\hline
\end{tabular}


country was not very straightforward. The trickiest case was the United States, where individual states have their own divorce laws (Glendon, 1987, 1989; Nakonezny et al., 1995; Friedberg, 1998). Because we could not distinguish between different states, we treated the United States as a single case. ${ }^{6}$

\section{Welfare state}

Welfare states are measured with two expenditure variables, social expenditure per GDP (general size of the welfare state) and family cash benefits per GDP (more targeted expenditure). For most countries, we were able to construct good time series of the developments of these social policy measures with data from the ILO $(1967,1988)$ and the OECD (1997). The exception was Poland, for which we found data for only one point in time.

\section{Values}

We include two variables reflecting the value environment: the percentage of denounced atheists to measure (non)religiosity (Barrett et al., 2001) and the national mean of a 10-point scale question enquiring whether one finds divorce justifiable or not, from the World Values Study $(1981 ; 1990 ; 1995)$ and the European Values Study (for Greece) (1999). For the former variable, the first data were found from 1970 onwards (projected back for earlier periods), for the latter, mainly from 1981 only.

\section{Family practices}

We measure family practices with a single time-dependent variable of 'unconventional family practices', which is a sum measure of the percentage of extra-marital births, the share of divorces per 100 marriages, and the percentage of 25-year olds who have ever lived in a consensual union (OECD 2002; Council of Europe 2000; FFS standard country tables http://www.unece.org/ead/pau/ffs/ f_h_151b.htm). These variables were strongly correlated $(0.7-0.8)$, and therefore, they were combined to proxy the social costs and innovativeness of divorce and the 'conventionality' of the family institution.

\section{Modernity and the labour market}

We use three variables as indicators of female labour market conditions and 'modernity': the degree of urbanization, the percentage of employment in the service sector, and the percentage of economically active women (World Bank World Development Indicators, ILO Labour statistics http://laborsta.ilo.org/, and United Nations Common Database http://unstats.un.org/unsd).

\section{Models}

\section{Within-Country Discrete-Time Event- History Models}

In our first analyses, we model the effects of female education on the risk of union disruption separately in each country with discrete-time event-history analysis techniques (Yamaguchi, 1991). Event-history analysis regresses the conditional probability of experiencing an event at time $t$ (union disruption)—providing that it has not happened before-on selected covariates, as described above. Data subsequently transformed to discrete-time format are analysed with logistic regression models using STATA 8.2 software.

\section{Multilevel Models}

We use multilevel discrete-time event-history models to test for the effects of the macro-variables. Replacing countries with variables is generally regarded as a valuable strategy in comparative research (e.g. Przeworski and Teune, 1970). Here, we deal with an additional dimension, historical time, and therefore, we replace country at a specific period (measured by union cohort in the within-country models) with direct measures of divorce legislation, social policies, values, demographical practices, and the level of the economy. To analyze these data, we restructured the data file into a two-level data structure: duration of the first marriage (organized in person-years) and countries at a particular point in time (Hox, 2002).

The models include the education dummies, the duration terms, parental divorce, marriage cohort, interactions between education and marriage cohort, the main effect of the macro-variables, and the interactions between the macro-variables and education. Similar to the discrete-time event-history analyses in the previous section, the data are analyzed with multilevel logistic regression models (cf. Hox, 2002). The models were estimated using MLwiN (Rasbash et al., 2000).

\section{Results from Event-History Analyses}

To get an overview of the relationship between female education and divorce in the 17 countries, Table 3 summarizes the main effects of education. We estimated two models for each country. The first model (Model A) is a reduced-form model, with education, linear, and squared terms of marital duration, year of marriage, and parental 
divorce (except for Norway). This model, including a minimal number of control variables, is used to estimate the 'gross' effect of education. The second model (Model B) adds age at marriage, pre-marital cohabitation (except for Estonia), and pre-marital birth. These variables are not control variables in the strict sense, because they are also affected by education. However, because these variables are often included in models on divorce risks, we estimated this model to estimate a more conservative 'net' effect for education, after adjusting for the three important intervening variables. The estimates of the control variables are mainly in line with what would be expected on the basis of the existing literature, with pre-marital cohabitation, pre-marital children, and parental divorce showing a positive effect, whereas the coefficient for age at marriage is negative.

As to the effects of education, Table 2 summarizes two main results of interest for this article. First, the effect of education on divorce varies across countries, and second, this result is rather robust with regard to model specification.

According to the A Models, women with high or medium levels of education have lower divorce risks than poorly educated women in Austria, Lithuania, and the United States. Furthermore, highly educated women have lower divorce risks than women with low or medium education in Flanders. In France and Spain, women with medium education divorce more than women with low education; in Greece, highly educated women stand out with higher divorce risks; and in Italy and Poland, both educational groups divorce more than the poorly educated. For the other countries, we do not find statistically significant differences.

The inclusion of the three additional variables in the B Models generally moves the point estimates in a more positive direction. This changes the results for some countries. The negative and significant effects of high education in the A Models for Flanders and the United States become non-significant, and the same estimate for the Norwegian model becomes positive and significant. The positive estimates in Model A generally become stronger (France, Greece, Italy, Poland, and Spain), while the negative Austrian and Lithuanian estimates do not change significantly. These results can be interpreted as showing that women with less education are at the aggregate level more likely to engage in behaviours that increase the risk of divorce. According to more detailed analyses of the changes in the point estimates (not shown, available on request), women with less education marry younger and are usually more likely to have a child out of wedlock, whereas women with higher education levels more frequently cohabit before marriage.

The estimates in most cases are different in Model B as compared with Model A, thus, in some countries, changing the conclusions on the effect of education on divorce. More importantly for this article, however, differences between the countries remain. Adding the three variables does not explain the differences between countries in the educational gradient of divorce. The country differences are mainly as one would expect on the basis of the literature and substantive knowledge of the countries. The most surprising result is perhaps the negative gradient in Austria. The non-negative gradients in the Nordic countries do not conform to results from many previous studies (cf. Hoem, 1997; Jalovaara, 2003; Lyngstad, 2004).

In Table 3, we test whether the educational gradients have changed over time. We insert interaction terms between the year of marriage and the education dummies into Model A of Table 2 and compare the models with regular chi-square tests. We chose to use the reduced-form equation to boost statistical power, especially given the limited number of events in some countries. However, the results from these models were very similar to those from models including the three intervening variables (not shown). In a manner similar to the main effect models, the inclusion of the intervening variables made the main effects more positive and weakened the statistical significance of the model fit statistic in Italy and, to a greater degree, in the United States. For the most part, however, the interaction effects remained very similar. This suggests that the change in the educational gradient of divorce is not exerted through the intervening variables. The results in the fifth column of Table 3 show the differences in the chi-squares and the degrees of freedom between the models. We can see that the inclusion of the interaction terms improves the model's fit for Flanders, Finland, Hungary, Lithuania, Poland, Sweden, and the United States. In France and Italy, the model including the interactions provides a better fit, which is significant at the 10-per cent level. In these nine countries, thus, there has been a change in the relationship between female education and divorce risk. ${ }^{7}$

Columns six to ten report the parameter estimates of the main effects and the interaction terms of the year of marriage and education. They show that, as predicted by our second hypothesis, all the significant interactions have a negative sign. ${ }^{8}$ In Finland, France, Hungary, Poland, and Sweden, the risk of divorce for those with high or medium levels of education decreased relative to the less educated, whereas in Flanders, Italy, and the 
ڤั

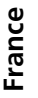

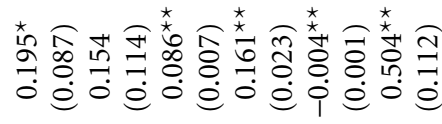

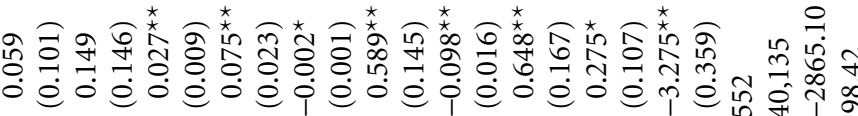

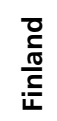

范

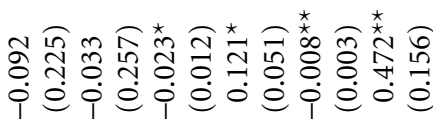

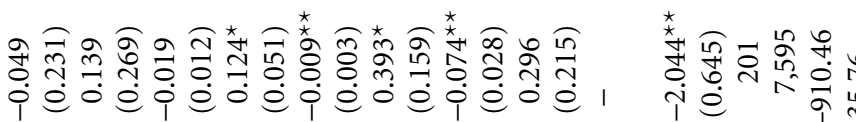

ñ.

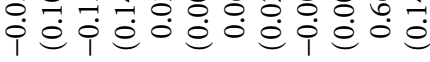

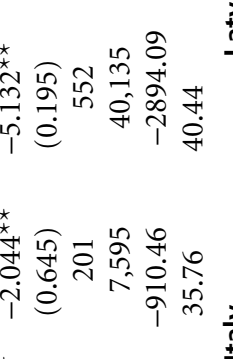

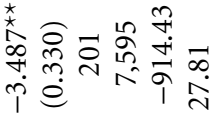

좊

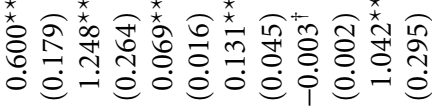

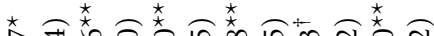

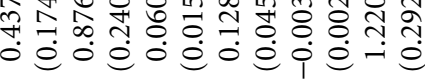

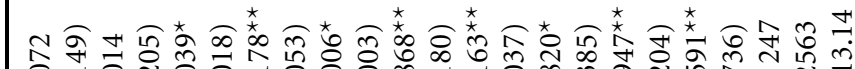

$\frac{\frac{n}{4}}{\frac{0}{0}}$

:

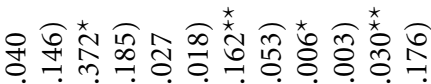

i $\dot{e}$ i

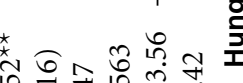

î̉

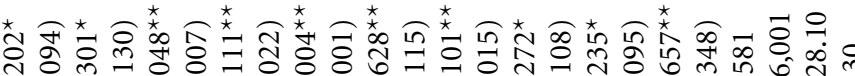

竞

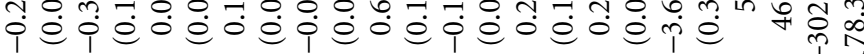

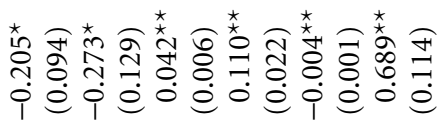

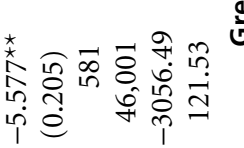

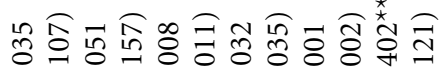

i

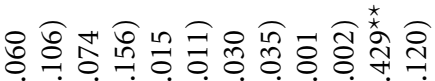

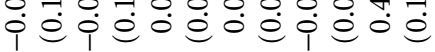

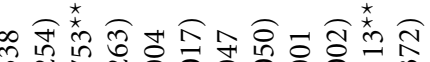
仓

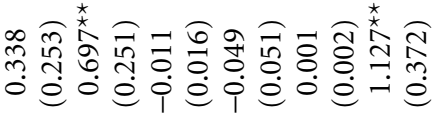

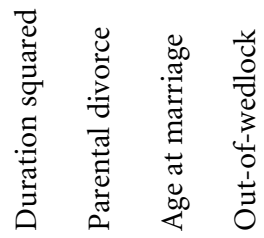

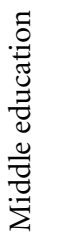

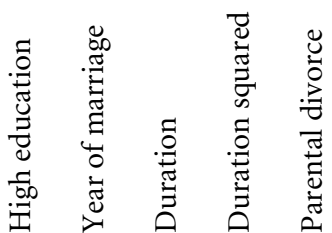




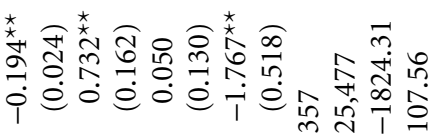

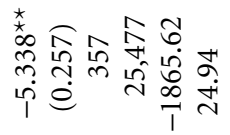

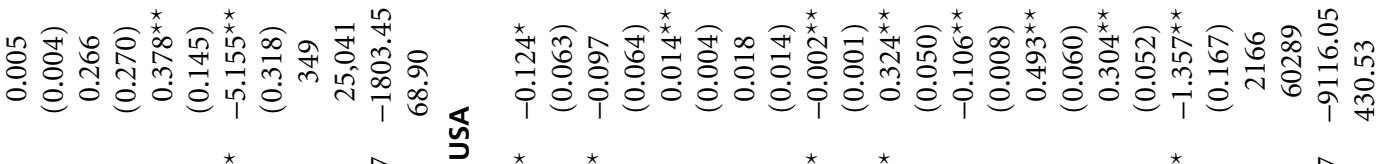

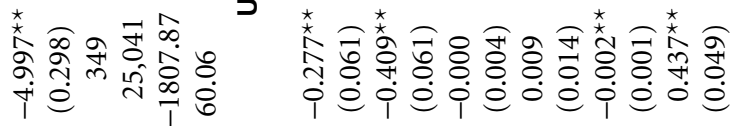

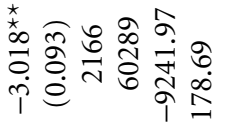

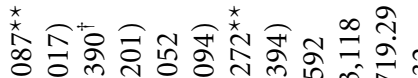

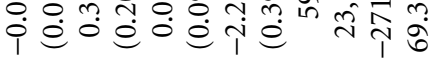

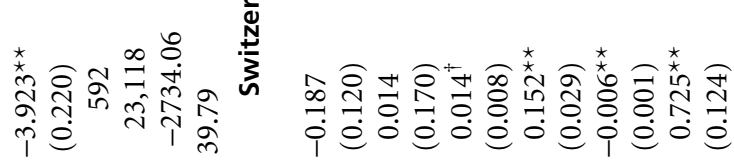

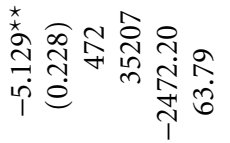

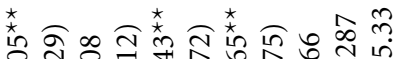
i.

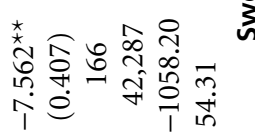

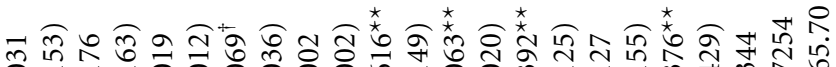
:

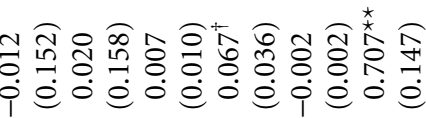

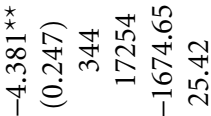

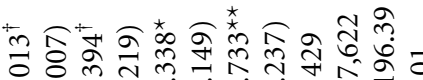
:

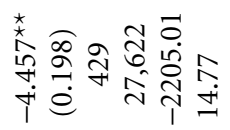

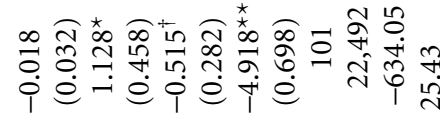

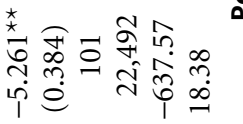

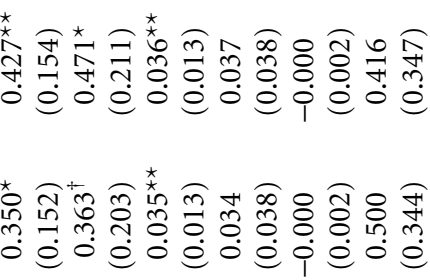

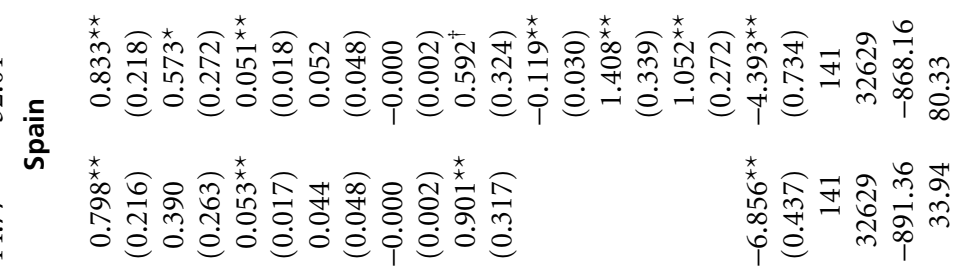

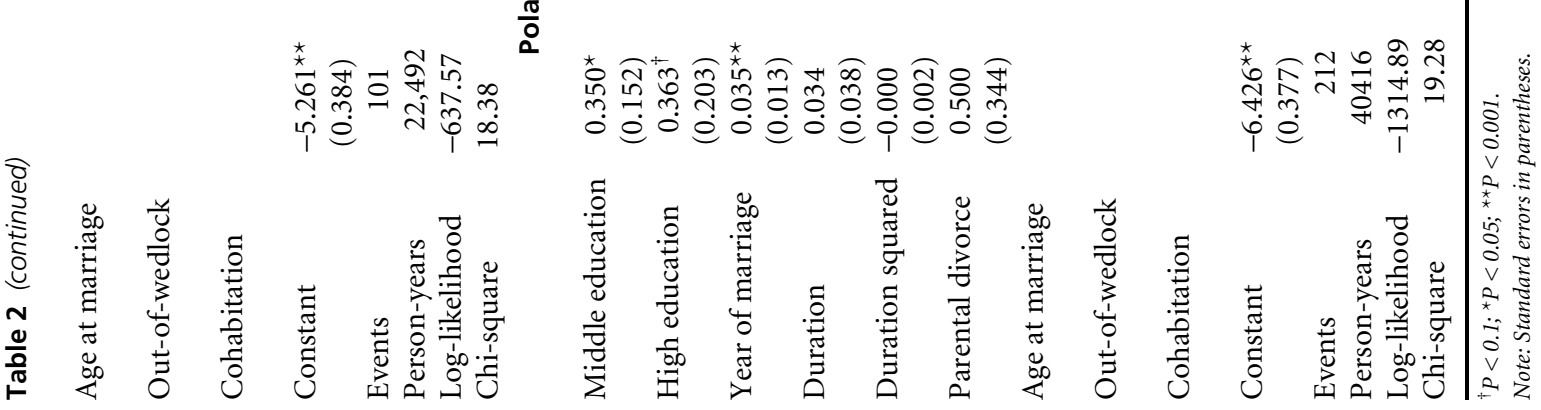




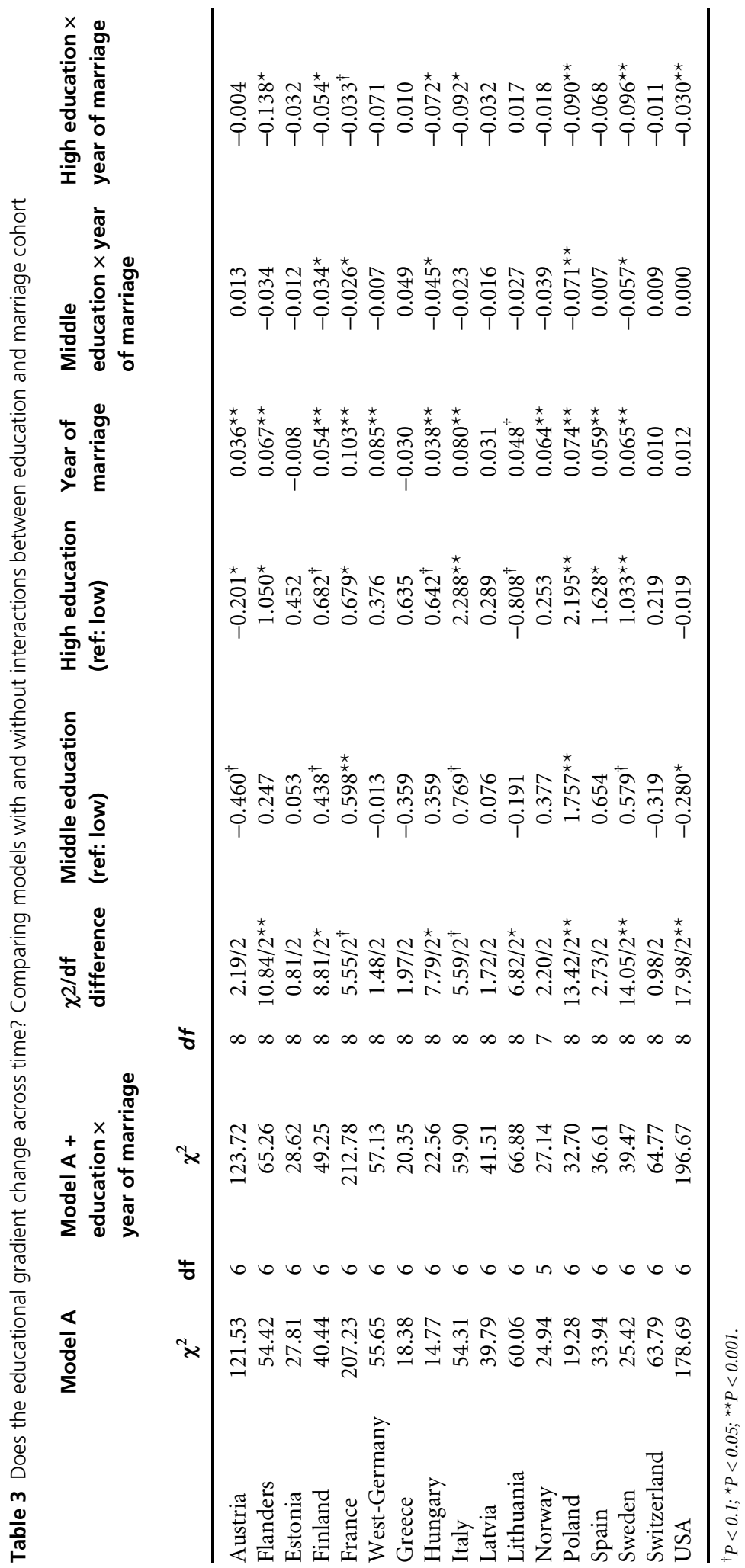


United States, there was a decrease only among the highly educated.

The results suggesting a change in Finland and the United States contradict previous results by Härkönen (2005) and Teachman (2002) but are in line with those by Raley and Bumpass (2003). However, as mentioned above, these studies used different model specifications, and in the case of the latter two, different time periods. This also explains the conflicting results between Table 2 and some Finnish and Swedish studies (Hoem, 1997; Jalovaara, 2003). In Finland and Sweden, the educational gradient of divorce was clearly positive in the oldest marriage cohorts (see also Blossfeld et al., 1995) but became more negative later. The cross-cohort differences thus cancel each other out.

In conclusion, the results in Table 3 support our second hypothesis of a change towards a more negative educational gradient of divorce in nine of our 17 countries.

\section{Results from Multilevel Event-History Analyses}

Table 4 summarizes the results from our first multilevel analyses with the data pooled over all countries. ${ }^{9}$ Model A includes the micro-variables and the interactions between education and marriage cohort. As expected, the interaction terms between year of marriage and education are negative and significant, pointing to an increasingly lower education gradient of divorce at the aggregate level of the 16 countries. ${ }^{10}$ As seen from the bottom row, significant between-country variance remains.

Next (Models B-J), we add each macro-variable and its interaction with the education dummies separately to Model A. This is the first step in examining how specific macro-level factors are associated with the differences in the educational gradient of divorce. The main effects of the macro-variables are in most cases significant, while the estimates of the micro-variables remain considerably stable. However, we are more interested in the coefficients of the interaction terms and the changes in the interaction between the year of marriage and education.

Examining the interaction terms between the macrovariables and education, we can see that divorce legislation and the social value variables are not associated with cross-national and temporal differences in the educational gradient of divorce. At the same time, we find that more favourable female labour markets (measured with service employment and female labour market activity) and more unconventional family practices are associated with a more negative educational divorce gradient, as expected on the basis of the third hypothesis. Urbanization is also related to lower rates of divorce among women having completed higher education. Higher social spending, and overall social expenditure in particular, is associated with a more positive educational gradient. When we look at changes in the interaction terms between education and marriage cohort, we can see that the value of the term changes by more than the standard error only in Models G (unconventional family types) and I (employment in services).

Table 5 continues the analyses by combining some of the macro-variables in the same model. Due to a limited number of countries, we chose only the most important variable from each group, based on the significance and strength of the interaction terms. The model thus includes social expenditure, unconventional family types, and the percentage of economically active women. The model including all three macro-variables and their interactions with educational attainment failed to converge. Therefore, Models $\mathrm{L}-\mathrm{N}$ include the three different combinations of two variables.

First, Model K shows the main effects of the three macro-variables and the micro-variables. The effect of social expenditure is positive, while, surprisingly enough, that of female economic activity is negative. The main effect of unconventional families is non-significant. When we include unconventional family types and female economic activity, along with their interactions with education in the same model (Model L), the interaction between female economic activity and high education becomes non-significant, while that between female economic activity and medium levels of education is barely significant at the 10 per cent level. ${ }^{11}$ On the contrary, the parameters for the interactions between unconventional families and education remain significant (and negative). In the other two combinations (social expenditure and unconventional families, social expenditure, and female activity), all the interactions remain significant. The signs of the significant interactions are the same as in Table 4. The negative relationship between the educational gradient and the higher female labour market activity is as expected (Model N), whereas the finding that higher social expenditure is still related to a more positive educational gradient contradicts our third hypothesis.

On the basis of the multilevel models, we conclude that many of the macro-variables have no significant effect on the educational gradient of divorce. Two societal factors stand out, however. A change in the family institution towards more liberal and unconventional 


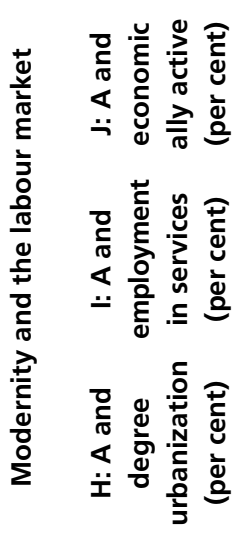

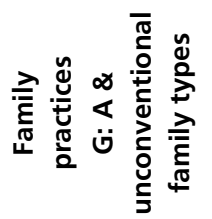
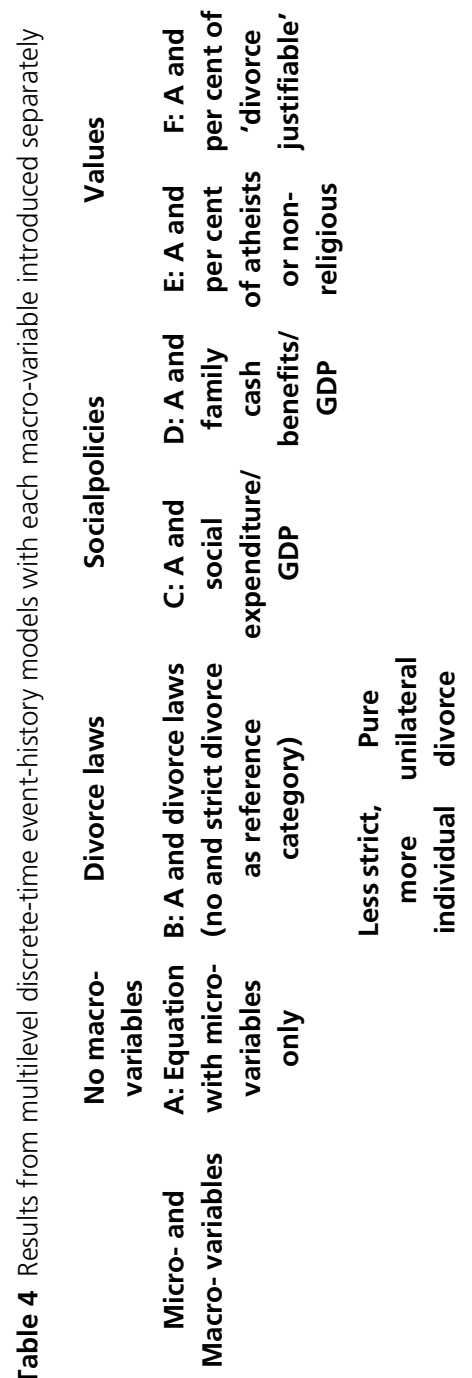

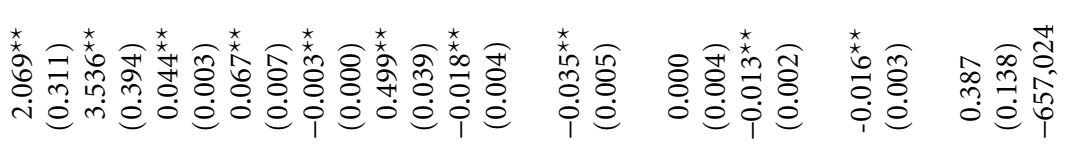

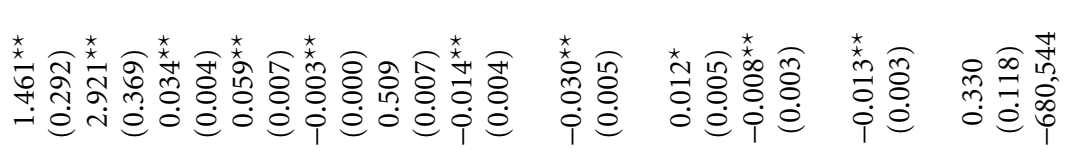

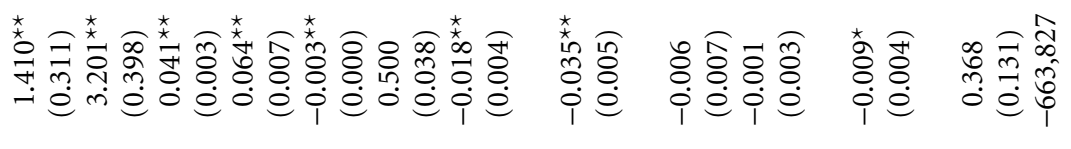

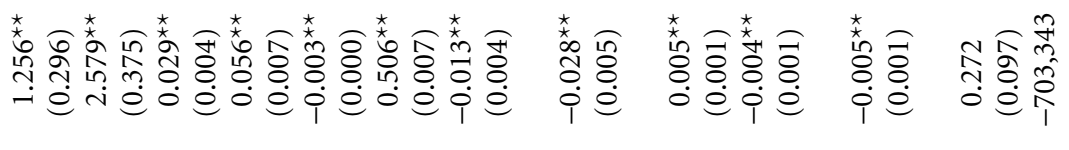

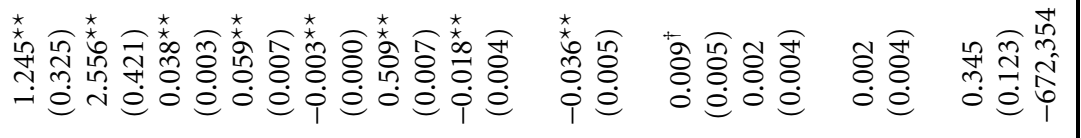

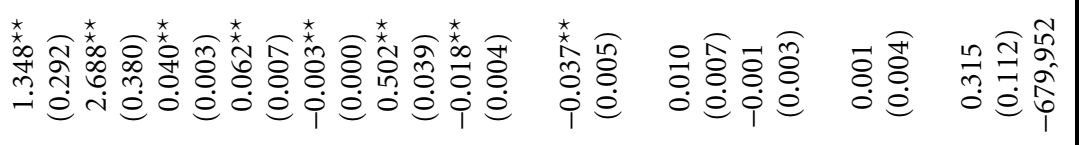

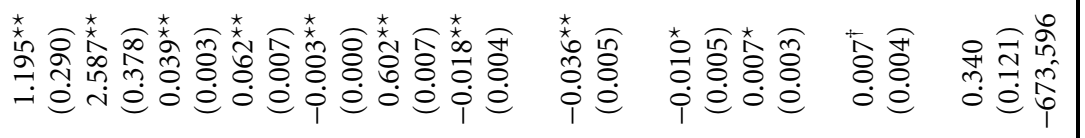

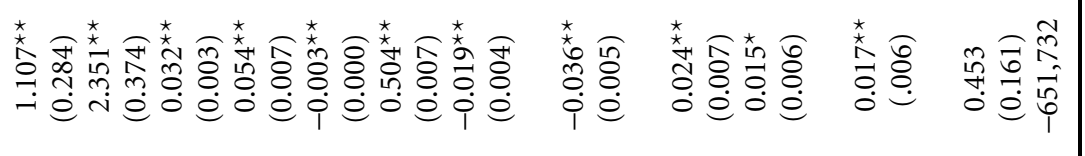

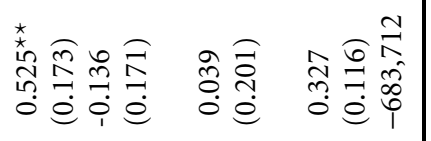

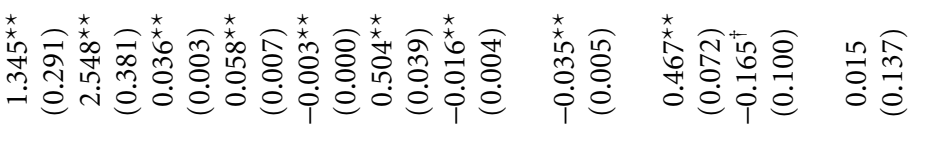

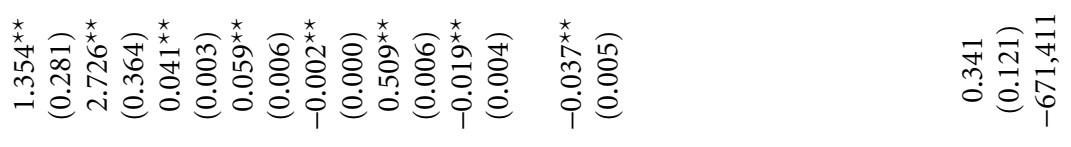

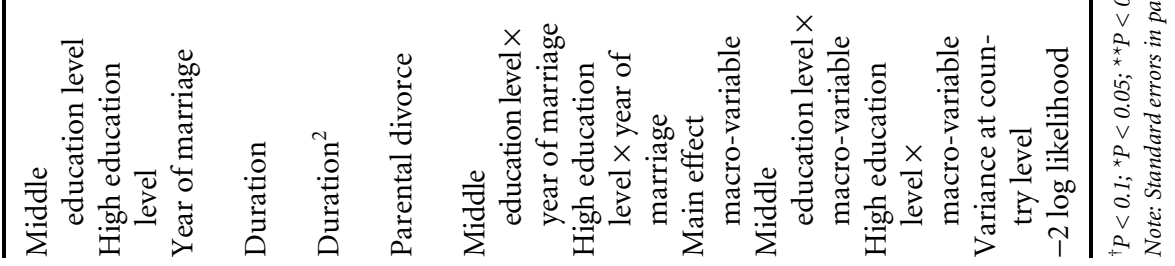




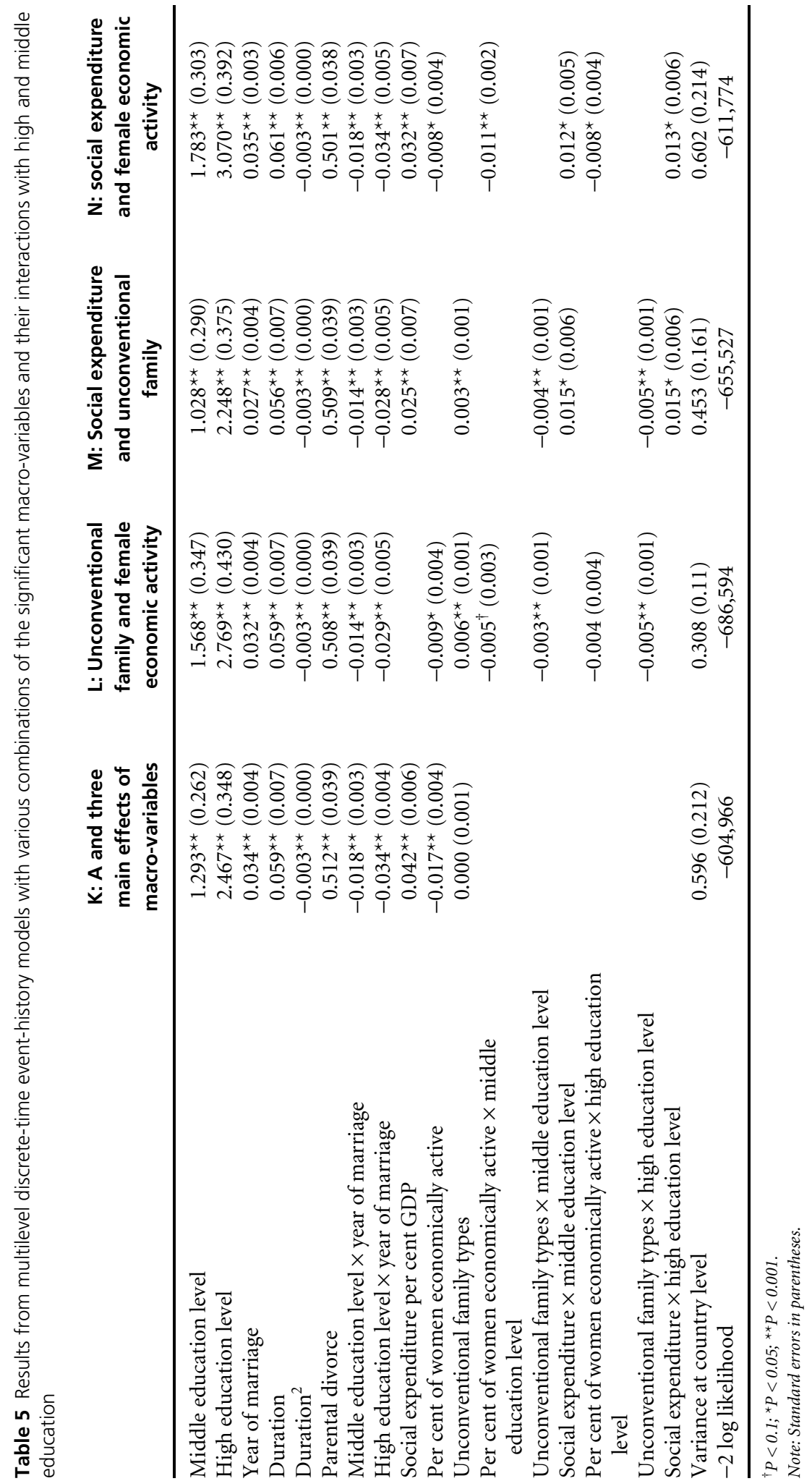


practices (divorces, out-of-wedlock births, and cohabitation) is associated with a more negative educational gradient of divorce, whereas a more generous welfare state (proxied by social expenditure per capita) is related to a more positive gradient.

\section{Conclusions and Discussion}

In this article, we have examined the relationship between female educational attainment and the risk of divorce across marriage cohorts in 16 European countries and the United States with data from the FFS. Beginning with a hypothesis by William J. Goode (1962), we expected that this effect varies across countries and that the effect of education on divorce would become more negative over time. We also aimed to link this variation to cross-national and cross-temporal differences in the legal, social, and economic costs of divorce, measured by the strictness of divorce legislation, social policies, values, family practices, and social and labour market conditions.

After estimating event-history models separately for each country, we found that overall the educational gradient of divorce was positive in France, Greece, Italy, Poland, and Spain and negative in Austria, Flanders, Lithuania, and the United States. In the other countries, statistically significant differences were not found. We also found that, in line with our second hypothesis, the educational gradient became more negative in Flanders, Finland, France, Hungary, Italy, Lithuania, Poland, Sweden, and the United States. In the other countries, no changes were found.

The overall country differences in the educational gradients seem to support Goode's hypothesis of a positive association between the costs of divorce and its educational or class gradient. The countries found to have positive gradients were as expected, although the negative gradients in Austria and Flanders can be regarded as somewhat surprising. Goode also made a farsighted hypothesis of a change towards a more negative educational gradient of divorce, a pattern found in nine of the 17 countries.

Our further analyses of the relationship between the educational gradient of divorce and the direct measures of its legal, social, and economic environment point to two factors of particular importance. First, a change towards a less marriage-centred family institution and more unconventional family practices is associated with faster growth in divorce rates in the lower strata of society. This can be interpreted as meaning that the high social costs (in terms of sanctions, innovativeness, and so on) of divorce demand resources if they are to be overcome. These same resources can be helpful in establishing and maintaining a stable marriage when these costs are low. From this point of view, however, it was surprising to find no relationship between the strictness of divorce legislation and the educational gradient (cf. Goode, 1970: 85-86). Second, we found that welfare state generosity is related to a more positive educational gradient of divorce. This is contrary to our hypothesis. This may suggest that welfare state generosity helps in stabilizing marriages amongst those with less human capital and more economic-based marital strain (cf. Goode, 1962; Oppenheimer, 1997; Jalovaara, 2003). We also found a link between increasing female labour market opportunities and a negative educational gradient. This, however, disappeared when we controlled for unconventional family types and this variable's interaction with education, suggesting that the social and cultural aspects of family change are more important than the closely related female labour market possibilities.

Needless to say, more research is needed to test the robustness of these findings and interpretations. While we have been able to establish cross-national variation in the partial correlation between female education and divorce, further research on the changes in the educational gradient-with larger national data sets-would be welcomed. Furthermore, we are able to point only tentatively to some factors behind these differences and trends. In this sense, the situation is similar to the research on the increasingly negative educational gradient of single motherhood in the United States (Ellwood and Jencks, 2004). The cross-country differences may also reflect other things, such as different patterns of selection in education and marriage. ${ }^{12}$ Moreover, we acknowledge the possible restrictions posed by the data and the macro-variables in particular. Nevertheless, however tentative they may be, we do think that our results point to important factors behind the differences across time and between countries. Further research is needed to test the hypotheses of the factors shaping the educational gradient of divorce. We also agree with the concerns of cumulative disadvantage (family and labour market) among the less educated and their families (McLanahan, 2004). Even though some of the factors behind these differential trends may be hard to change, our results suggest that public policy can play a role in equalizing the chances for stable families and the benefits associated with them. 


\section{Notes}

1. The processes affecting higher order marriages are different from those affecting first marriages (e.g. Martin and Bumpass, 1989). Men were excluded due to the few cases in the data.

2. Because in some countries (Italy, Poland, Greece, and Spain) there were only a few divorces in the early periods, we did not censor durations of more than 10,15 , or 20 years. However, the models with a censoring on the 15th year of the union (if still intact) gave very similar results (not shown). We also coded death and 'forced living apart together' as censored. A competing-risks analysis with these categories did not change our results.

3. Year of marriage was recoded to start from 0.

4. Following Thornton and Rodgers (1987) and Teachman (2002), we also tested whether the risk of divorce at different durations varied across the marriage cohorts. In a model with the main and squared effect of duration, and an interaction between duration and marriage cohort (not shown), we did not find stability of dissolution risk at different durations across cohorts. We thus conclude that, if we disregard the assumption of a strong interaction effect between marriage cohort and duration, our data support the hypothesis of period effects instead of cohort effects.

5. Using a direct period measure would have been inconvenient, because in Italy, Spain, Greece, and Poland, the number of divorces in the early periods was too small to permit sustainable analyses.

6. We used the year 1970 (when California was the first state to enact no-fault legislation) as a breakpoint between categories 1 and 2, and the year 1985 (when South Dakota was the last state to enact nofault divorce) as a breakpoint in a move from category 2 to category 3 . This solution admittedly provides only an approximation, but because 'divorce tourism' between states was possible (Castles and Flood, 1993) and because the law in many cases was a dead letter with pressures from neighbouring states, we regard this as a second-best option.

7. Due to the limited number of events in some countries, the data might not reveal some actual changes.

8. Most of the non-significant coefficients also have a negative sign.

9. Norway is excluded, because information on parental divorce is not available.

10. We also confirmed the findings from the previous section concerning significant variation in the effects of education with random coefficient models (not shown).

11. We also tested whether replacing female economic activity with percentage of employment in services as a measure of female labour market opportunities would change the results (not shown). The interaction terms between service employment and education were both non-significant, whereas estimates of the interactions between unconventional family types and education were the same as in Model L.

12. In our companion paper, we compared results from first marriages to results from all first unions (Härkönen and Dronkers, 2006). As a general conclusion, the results were remarkably similar, thus suggesting no effects of differential selection to marriage.

\section{Acknowledgments}

Previous versions of this article were presented in Cologne, Princeton, San Francisco, Southampton, and Stockholm. The authors thank the participants in these conferences and seminars and Gunnar Andersson, Hans-Peter Blossfeld, Tak Wing Chan, Paul de Graaf, Matthijs Kalmijn, Torkild Hovde Lyngstad, Anne-Rigt Poortman, and Michael Wagner for valuable comments. The research was supported by a grant from the Academy of Finland (no. 205226).

\section{References}

Allison, P. D. (1995). Survival Analysis Using the SAS System. A Practical Guide. Cary (NC): SAS Publishing.

Amato, P. (1996). Explaining the Intergenerational Transmission of Divorce. Journal of Marriage and the Family, 58, 628-640.

Andersson, G. and Philipov, D. (2002). Life-Table Representations of Family Dynamics in Sweden, Hungary, and 14 Other FFS Countries: A Project of Descriptions of Demographic Behaviour, Demographic Research, 7, 67-144.

Barrett, D. B., Kurian, G. T. and Johnson, T. M. (2001). The World Christian Encyclopedia: A Comparative Survey of Churches and Religions in the Modern World. Part 1. Oxford: Oxford University Press.

Becker, G. S. (1981). A Treatise on the Family. Cambridge, MA: Harvard University Press.

Berrington, A. and Diamond, I. (1999). Marital Dissolution Among the 1958 British Birth Cohort: The Role of Cohabitation. Population Studies, 53, 19-36.

Blossfeld, H., de Rose, A., Hoem, J. M. and Rohwer, G. (1995). Education, Modernization, and the Risk of 
Marriage Disruption in Sweden, West Germany, and Italy. In Oppenheim Mason, K. and Jensen, A. (Eds), Gender and Family Change in Industrialized Societies. Oxford: Clarendon Press, pp. 200-222.

Boschan, S. (1972). Europäisches Familienrecht. München: Verlag Franz Vahlen.

Bumpass, L. L. and Sweet, J. A. (1972). Differentials in Marital Instability: 1970. American Sociological Review, 37, 754-766.

Castles, F. G. and Flood, M. (1993). Why Divorce Rates Differ: Law, Religious Beliefs, and Modernity. In Castles, F. G. (Ed.), Families of Nations. Patterns of Public Policies in Western Democracies. Aldershot: Dartmouth, pp. 293-326.

Chan, T. W. and Halpin, B. (2005). The Instability of Divorce Risk Factors in the UK. Working Paper No. 2005-06, Oxford: Department of Sociology, University of Oxford.

Chester, R. (1977). Divorce in Europe. Leiden: Martinus Nijhoff.

Chloros, A. G. (1978). The Reform of Family Law in Europe. Deventer: Kluwer.

Council of Europe (2000). Recent Demographic Trends in Europe. Strasbourg: Council of Europe.

Diekmann, A. and Klein, T. (1991). Bestimmungsgründe des Ehescheidungsrisikos. Eine Empirische Untersuchung mit den Daten des Sozioökonomischen Panels. Kölner Zeitschrift für Soziologie und Sozialpsychologie, 43, 271-290.

Dronkers, J. (2002). Bestaat er een Samenhang Tussen Echtscheiding en Intelligentie? Mens en Maatschappij, 77, 25-42.

Ellwood, D. T. and Jencks, C. (2004). The Uneven Spread of Single-Parent Families: What Do We Know? Where Do We Look for Answers? In Neckerman, K. (Ed.), Social Inequality. New York: Russell Sage Foundation, pp. 3-77.

Friedberg, L. (1998). Did Unilateral Divorce Raise Divorce Rates? Evidence from Panel Data. American Economic Review, 88, 608-627.

Glendon, M. A. (1987). Abortion and Divorce in Western Law. Cambridge, MA: Harvard University Press.

Glendon, M. A. (1989). The Transformation of Family Law. State, Law, and Family in the United States and Western Europe. Chicago: The University of Chicago Press.

Goode, W. J. (1962). Marital Satisfaction and Instability. A Cross-Cultural Class Analysis of Divorce Rates. In Bendix, R. and Lipset, S. M. (Eds), Class, Status, and Power. Social Stratification in Comparative Perspective. New York: The Free Press, pp. 377-387.

Goode, W. J. (1970 [1963]). World Revolution and Family Patterns. New York: The Free Press.

Goode, W. J. (1993). World Changes in Divorce Patterns. New Haven: Yale University Press.
Hamilton, C. and Perry, A. (2002). Family Law in Europe. London: Butterworths.

Hoem, J. M. (1997). Educational Gradients in Divorce Risks in Sweden in Recent Decades. Population Studies, 51, 19-27.

Hox, J. J. (2002). Multilevel Analysis. Techniques and Applications. Mahwah, NJ: Lawrence Erlbaum Associates.

Härkönen, J. (2005). Divorce Risk Factors Across Finnish Marriage Cohorts, 1954-1989. Yearbook of Population Research in Finland, 41, 151-164.

Härkönen, J. and Dronkers, J. (2006). Stability and Change in the Effects of Female Educational Attainment and the Risk of Union Disruption in Seventeen Countries. Working Paper No. 2006/04, Florence: Department of Political and Social Sciences, European University Institute.

ILO (1967). The Cost of Social Security. Geneva: International Labor Office.

ILO (1988). The Cost of Social Security. Geneva: International Labor Office.

Jalovaara, M. (2003). The Joint Effects of Married Partners' Socioeconomic Positions on the Risk of Divorce. Demography, 40, 67-81.

Kalmijn, M., de Graaf, P. M. and Poortman, A. (2004). Interactions Between Cultural and Economic Characteristics of Divorce in the Netherlands. Journal of Marriage and the Family, 66, 75-89.

Levinger, G. (1976). A Social Psychological View on Marital Dissolution. Journal of Social Issues, 32, 21-42.

Lobodzinska, B. (1983). Divorce in Poland: Its Legislation, Distribution and Social Context. Journal of Marriage and the Family, 45, 927-942.

Lutz, W., Wils, A. B. and Nieminen, M. (1991). The Demographic Dimensions of Divorce. The Case of Finland. Population Studies, 45, 437-453.

Lyngstad, T. H. (2004). The Impact of Parents' and Spouses' Education on Divorce Rates in Norway. Demographic Research, 10, 119-142.

Martin, T. C. and Bumpass, L. L. (1989). Recent Trends in Marital Disruption. Demography, 26, 37-51.

McLanahan, S. (2004). Diverging Destinies: How Children Are Faring Under the Second Demographic Transition. Demography, 41, 607-627.

Moskoff, W. (1983). Divorce in the USSR. Journal of Marriage and the Family, 45, 419-425.

Nakonezny, P. A., Shull, R. D. and Rodgers, J. L. (1995). The Effect of No-Fault Divorce Law on the Divorce Rate Across the 50 States and Its Relation to Income, Education, and Religiosity. Journal of Marriage and the Family, 57, 477-488.

OECD (1997). Social Expenditure Database. Paris: OECD.

OECD (2002). Social Indicators. Paris: OECD.

Ono, H. (1998). Husbands' and Wives' Resources and Marital Dissolution. Journal of Marriage and the Family, 60, 674-689. 
Oppenheimer, V. K. (1997). Women's Employment and the Gain to Marriage. Annual Review of Sociology, 23, 431-453.

Orloff, A. S. (1993). Gender and the Social Rights of Citizenship: The Comparative Analysis of Gender Relations and Welfare States. American Sociological Review, 58, 303-328.

Przeworski, A. and Teune, H. (1970). Logic of Comparative Social Inquiry. New York: John Wiley \& Sons.

Raley, R. K. and Bumpass, L. (2003). The Topography of the Divorce Plateau: Levels and Trends in Union Stability in the United States After 1980. Demographic Research, 8, 243-260.

Rasbash, J., Browne, W., Goldstein, H., Yang, M., Plewis, I., Healy, M., Woodhouse, G., Draper, D., Langford, I. and Lewis, T. (2000). A User's Guide to MLwiN. London: Multilevel Models Project.

Teachman, J. D. (2002). Stability Across Cohorts in Divorce Risk Factors. Demography, 39, 331-351.

Thornton, A. and Rodgers, W. J. (1987). The Influence of Individual and Historical Time on Marital Dissolution. Demography, 24, 1-22.
Wolfers, J. (2003). Did Unilateral Divorce Laws Raise Divorce Rates? A Reconciliation and New Results. Working Paper No. 10014, Cambridge, MA: National Bureau of Economic Research.

Yamaguchi, K. (1991). Event History Analysis. Newbury Park: Sage.

\section{Authors' Addresses}

Juho Härkönen, (to whom correspondence should be addressed), Department of Political and Social Sciences, European University Institute, Via dei Roccettini 9, San Domenico di Fiesole (Fl), Italy. Email: juho.harkonen@eui.eu.

Jaap Dronkers, Department of Political and Social Sciences, European University Institute, Via dei Roccettini 9, San Domenico di Fiesole (Fl), Italy. Email: jaap.dronkers@eui.eu.

Manuscript received: March 2006 